\title{
Photothermal Reactions of Nitrosobenzene and Halonitrosobenzenes in Solid-state
}

\author{
Anamarija Knežević, Tina Medančić, Srđan Milovac, Ivana Biljan, Ivan Halasz, and Hrvoj Vančik* \\ Department of Chemistry, Faculty of Science, University of Zagreb, Horvatovac 102A, 10000 Zagreb, Croatia
}

RECEIVED JUNE 1, 2010; REVISED NOVEMBER 25, 2010; ACCEPTED NOVEMBER 29, 2010

\begin{abstract}
Photothermal reactions of the dimers of nitrosobenzene, $m$-chloronitrosobenzene, and $p$ chloronitrosobenzene were studied in solid-state by IR spectroscopy at low temperatures and by X-ray powder diffraction. It was found for the first time that photothermal cycle (photolytic dissociation followed by thermal dimerization) could successively be performed also with $Z$-configured dimeric nitrosobenzene.

Halonitrosobenzenes dimers with $E$-configuration afforded different photo behavior depending on the position of halogen atom on the benzene ring: while $m$-halonitrosobenzenes do not dissociate under UV irradiation, $p$-chloronitrosobenzene, as well as previously studied $p$-bromonitrosobenzene photolyses very efficiently with recovering of the original crystal phase. Kinetics of thermal dimerization was measured in solid-state, and it was found that the reaction phase transformation occurs as a two-dimensional growth through the crystal. (doi: 10.5562/cca1714)
\end{abstract}

Keywords: nitrosobenzenes, photothermal reactions, solid-state

\section{INTRODUCTION}

While nitrosobenzenes appear in solution as equilibrium of two forms, monomers and dimers, in solid-state most of them crystallize predominantly in the form of dimers (azodioxides). ${ }^{1}$ In our previous work we have found that under cryogenic conditions in solid state, such azodioxides undergo photodissociation to corresponding nitrosobenzene monomers, which, in turn, redimerize quickly by warming above some critical temperature. ${ }^{2}$ Since these processes include breaking and rebinding the azodioxide nitrogen-nitrogen bonds, the system represents a sort of molecular OFF-ON switch, (Scheme 1).

The reaction mechanism of such a solid-state photodissociation of $p$-bromonitrosobenzene dimer was studied in detail by in situ X-ray diffraction analysis of the single-crystal-to-single-crystal transformation. ${ }^{3}$ Fast thermal redimerization above $170 \mathrm{~K}$ was explained as a consequence of a very close contact of neighboring Natoms in crystals of in situ prepared monomers. This non-covalent distance is even for $23 \%$ shorter than the sum of the van der Waals radii.

For possible application of such a molecular OFFON switch, the system must satisfy the condition that the dissociation-dimerization process is operative not only under cryogenic conditions, but also at room tem-

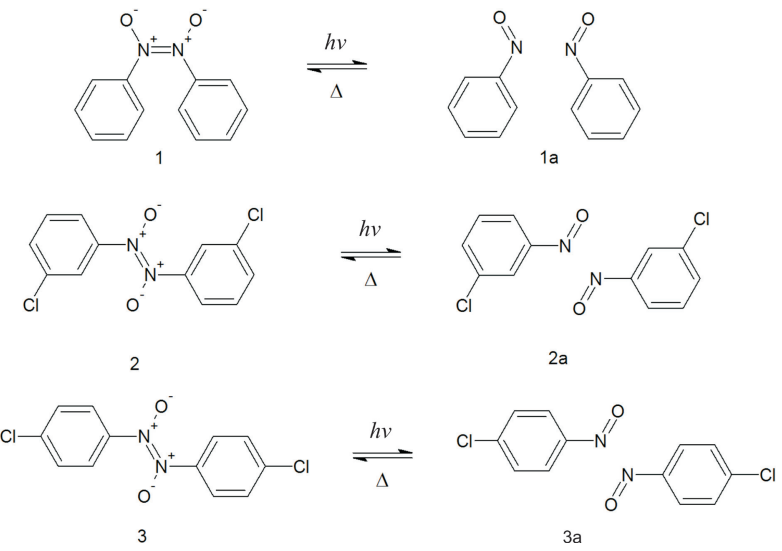

Scheme 1. Photothermal reactions of nitrosobenzene dimers.

perature. In this line it becomes necessary to search for more promising systems, which could afford such photothermal effect.

In this work we extend the investigation of reactivity, kinetics, as well as topochemistry of nitrosobenzene derivatives to $Z$-nitrosobenzene dimer $\mathbf{1}$, and $\mathrm{m}$ and $p$-chloronitrosobenzene, $\mathbf{2}$ and $\mathbf{3}$ (Scheme 1). Structure $\mathbf{1}$ is interesting because of its $Z$-stereochemistry. Namely, all the nitrosobenzene dimers previously studied by solid-state photochemistry were $E$ - stereomers.

* Author to whom correspondence should be addressed. (E-mail: vancik@chem.pmf.hr) 
Investigation of $\mathbf{2}$ and $\mathbf{3}$ is expected to provide new information about the effect of molecular packing on the photodissociation in the crystal lattice. Position of the chlorine atom on the benzene ring could have critical influence on the reaction path.

\section{EXPERIMENTAL}

Nitrosobenzene dimers were prepared by standard methods. ${ }^{4}$

Photolysis. A high-pressure Hg lamp $250 \mathrm{~W}$ was used for photolysis.

FT-IR kinetics. The solid-state dimerization rate was measured by following the temporal change in transmittance of the ONNO asymmetric stretching signal at $1258 \mathrm{~cm}^{-1}$ and the NO stretching signal of monomer at $1494 \mathrm{~cm}^{-1}$. Bruker Equinox FT-IR spectrometer with 1 $\mathrm{cm}^{-1}$ resolution was used for this measurement. The sample was prepared as a standard $\mathrm{KBr}$ pellet cooled by Leybold-Heraeus ROK 10 - 300 cyclic helium cryostat.

Powder diffractogram was taken on Philips PW 1840 diffractometer, Bragg-Brentan geometry, $2 \theta=5-35^{\circ}$.

\section{RESULTS AND DISCUSSION}

Nitrosobenzene dimer, 1. After photolysis of crystals of dimer 1 in $\mathrm{KBr}$ pellet at $23 \mathrm{~K}$ by a high-pressure $\mathrm{Hg}$ lamp, the signals at $1398 \mathrm{~cm}^{-1}$ and $1412 \mathrm{~cm}^{-1}$ assigned to symmetric and asymmetric stretching vibrations of

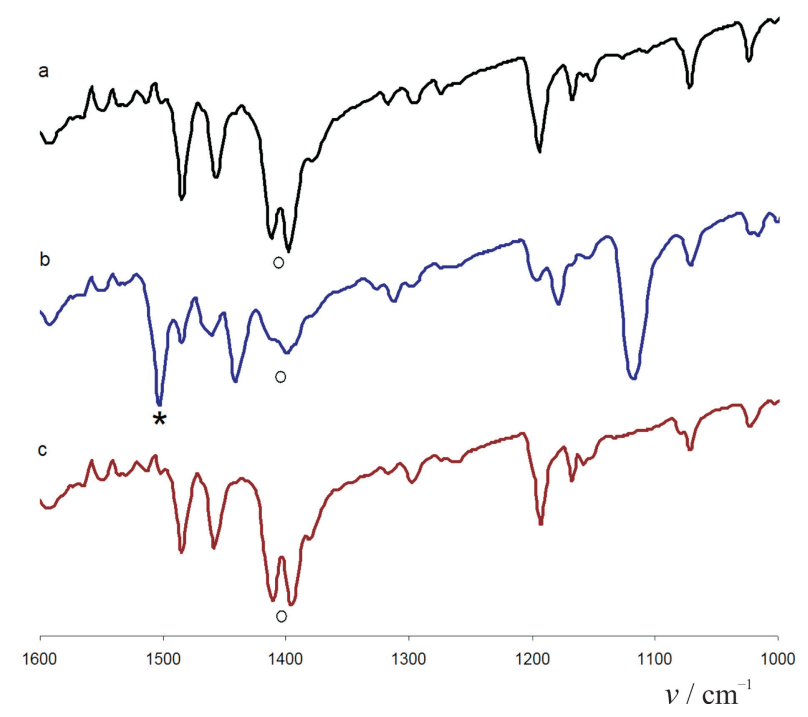

Figure 1. FT-IR spectrum of $\mathbf{1}$ in $\mathrm{KBr}$ pellet (a) at $23 \mathrm{~K}$, (b) after $30 \mathrm{~min}$ of photolysis at $23 \mathrm{~K}$ by a high pressure $\mathrm{Hg}$ lamp, (c) after warming to $300 \mathrm{~K}$. The signal characteristic for ONNO stretching vibration of dimer is labeled with $\mathbf{o}$, while the signal characteristic for $\mathrm{NO}$ stretching vibration of monomer is labeled with *. the Z-ONNO group strongly decreased in their intensity, and the new signals at $1503 \mathrm{~cm}^{-1}$, originated from $\mathrm{N}=\mathrm{O}$ stretching vibration of the nitroso monomer, appeared in the spectrum (Figure 1b).

By warming the sample to room temperature, the signals of the starting dimer were recovered, while the signal of the monomer disappeared (Figure 1c). Because the process can be visualized as changing the color from yellowish (dimer) to blue (monomer), the reaction is also an example of photochromic/thermochromic effect. The observed temperature of this thermal transition from monomer to dimer was between $265 \mathrm{~K}$ and $275 \mathrm{~K}$. The photolysis of $\mathbf{1}$ has been repeated at different temperatures, and it was found that the highest temperature at which this compound undergoes photochromic dissociation is $245 \mathrm{~K}$. This temperature is much higher than in the case of previously observed photoreaction of $p$-bromonitrosobenzene $(170 \mathrm{~K}){ }^{2}$

The transformation cycle that includes photodissociation followed by thermal redimerization was repeated five times. Relative intensity of the $1503 \mathrm{~cm}^{-1}$ band assigned to the monomer NO stretching vibration was more or less recovered after an each cycle (Figure 2). Evidently, this OFF-ON switching system has a high efficiency.

Chloronitrosobenzene dimers, $\mathbf{2}$ and $\mathbf{3}$. Irradiation of $m$-chloronitrosobenzene dimer $\mathbf{2}$ cooled in $\mathrm{KBr}$ pellet to $20 \mathrm{~K}$ did not afford any change in the IR spectrum. No dissociation was observed at any temperature between $15 \mathrm{~K}$ and the room temperature. The same experiment was made with $m$-bromo derivative of dimer 2 which also did not afford photodissociation. In contrast, dimer $\mathbf{3}$, in which the halogen atom is in para position, readily undergoes photodissociation at $25 \mathrm{~K}$. The effect was characterized by a disappearance of the IR signal at $1258 \mathrm{~cm}^{-1}$ assigned to asymmetric stretching vibration of the $E$-ONNO group, ${ }^{5}$ and the appearance of the signal characteristic for monomer NO stretching vibration at $1494 \mathrm{~cm}^{-1}$ (Figure 3). The thermal redimerization started at the temperature as high as $295 \mathrm{~K}$. Moreover, at

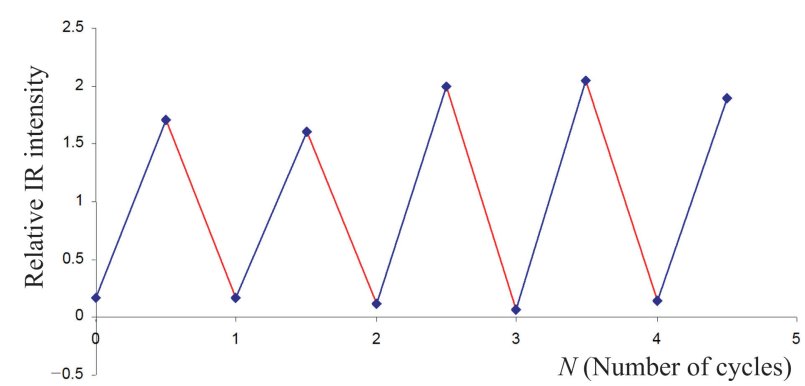

Figure 2. Cyclic change in the intensity of the band assigned to monomer NO stretching vibration $\left(1503 \mathrm{~cm}^{-1}\right)$ of $\mathbf{1}$ after successive photodissociations (blue line) and thermal dimerizations (red line). 


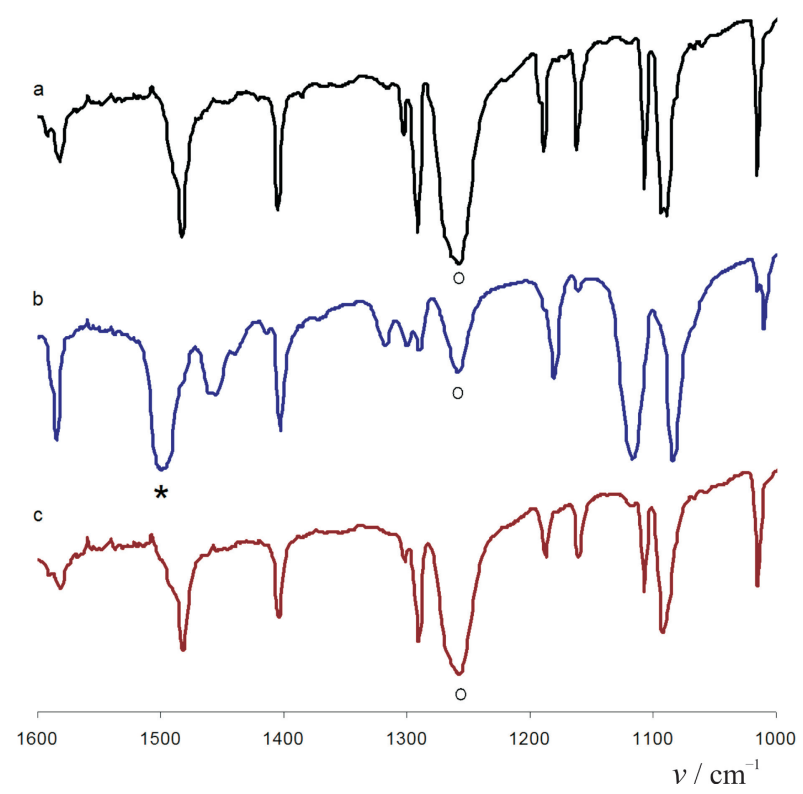

Figure 3. FT-IR spectrum of 3 in $\mathrm{KBr}$ pellet (a) at $20 \mathrm{~K}$, (b) after $50 \mathrm{~min}$ of photolysis at $20 \mathrm{~K}$ by a high pressure $\mathrm{Hg}$ lamp, (c) after warming to $300 \mathrm{~K}$. o labels dimer, * labels monomer.

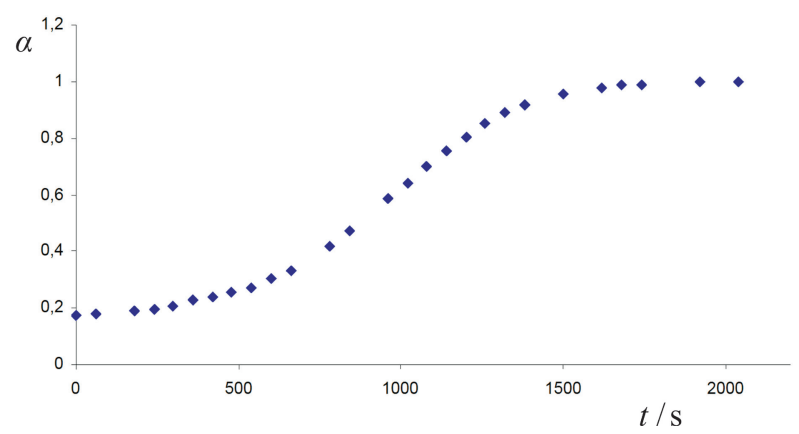

Figure 4. Kinetic curve of solid-state dimerization of $\mathbf{3}$ in $\mathrm{KBr}$ pellet at $295 \mathrm{~K}$.

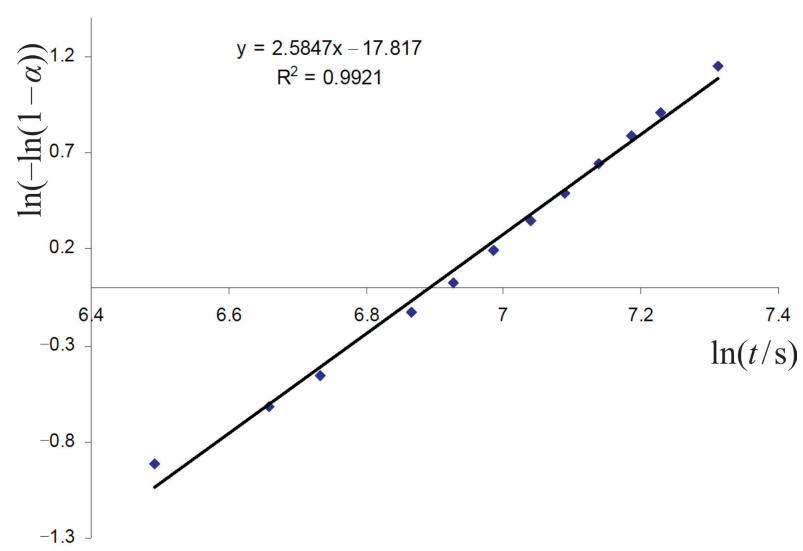

Figure 5. Linear Sharp-Hancock plot obtained from kinetics data for solid-state dimerization of $\mathbf{3}$. this temperature we have succeeded to measure kinetics of dimerization by following the intensity of the disappearing NO stretching band at $1494 \mathrm{~cm}^{-1}$. The obtained kinetic curve is a sigmoid that is typical for solid-state reactions and phase transformations (Figure 4). ${ }^{6}$ From the analysis of the reaction by using the standard Avrami-Erofeev model, the reaction in the crystal can be described as a two-dimensional growth of the product phase. ${ }^{6}$ Namely, linear plot of logarithms of the extent of the reaction, $(\alpha)$ versus logarithm of time (SharpHancock plot) gives the coefficient $m=2.58$ characteristic for the two-dimensional process (Figure 5).

Phase reversibility of the photothermal cycle. The powder X-ray diffractograms were recorded for polycrystalline samples before photolysis, and at the end of the cycle, i.e. after thermal redimerization. As it can be seen in Figure 6, the diffractograms do not differ, and it follows that the compound after photodissociation followed by redimerization recovers its origins crystal phase.

This finding supports the previous conclusions about the topochemical reversibility of such cycle, obtained from observations of the single-crystal-to-singlecrystal transformations. The hindered solid-state photodissociation of meta halogen derivatives ( 2 and 4 ) can be explained by formation of interhalogen bonds ${ }^{7}(\mathrm{Cl}---\mathrm{Cl}$, or $\mathrm{Br}---\mathrm{Br}$ ) in their crystal lattice (Figure 7). The $\mathrm{Br} . . \mathrm{Br}$ contacts are identified by typical interatomic distance of $3.76 \AA$. If such bonds are in meta-position, they can hinder the necessary partial rotation of benzene rings, which always accompanies dissociation. Oppositely, benzene rings have freedom to rotate partially and to enable dissociation if interhalogen bonds are formed between halogens in para-position (Figure 7). Note, that $\mathbf{2}$ and $\mathbf{4}$ are isostructural because they have analogous unit cell, which for $\mathbf{2}$ have parameters a $(\AA)$ 12.302(2), b

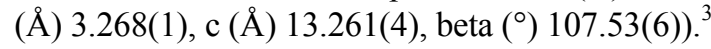

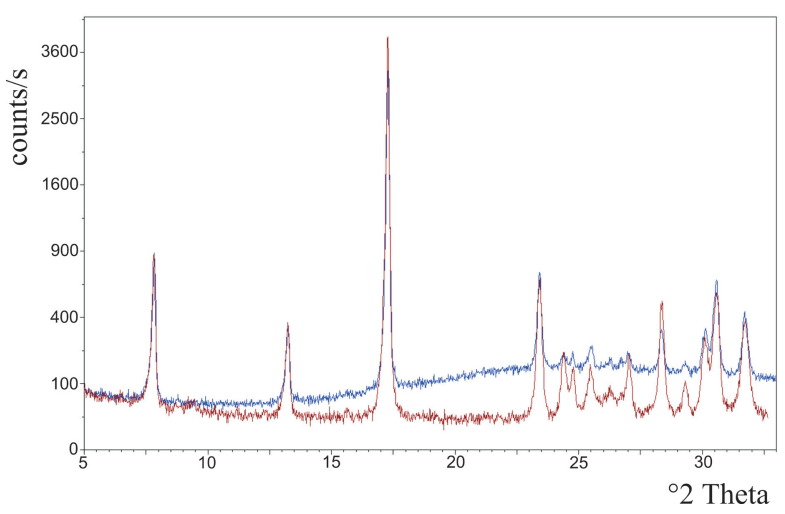

Figure 6. Powder diffractogram of $\mathbf{3}$ before (red line), and after the photothermal cycle (blue line). 

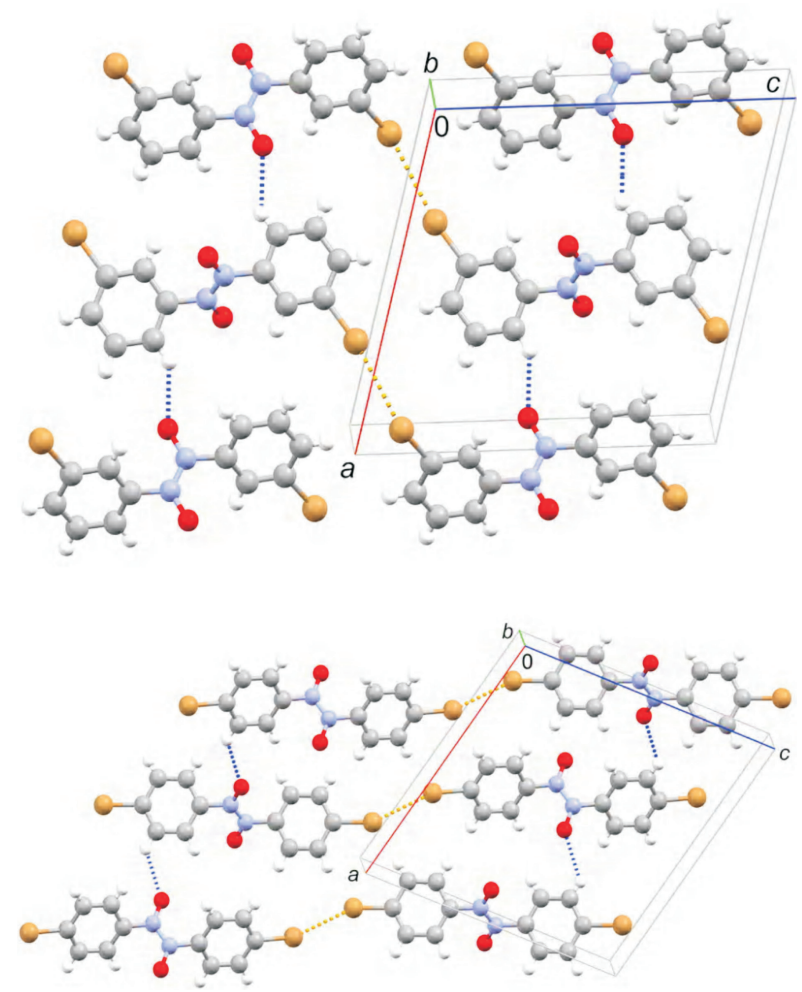

Figure 7. Interhalogen bonds ( $\mathrm{Br}----\mathrm{Br})$ in $m$ - (top picture) and $p$-bromonitrosobenzene (bottom).

\section{CONCLUSIONS}

Since in previous investigations only trans nitroso dimers were successfully photodissociated in the solidstate, here we found for the first time that the photothermal cycle (photolytic dissociation followed by thermal dimerization) could successively be performed also with $Z$-configured nitrosobenzene dimers and that the process occurs at much higher temperature than in previously studied $E$-derivatives.

In the second part of this work we studied the solid-state photothermal transformations of dimeric halonitrosobenzenes with $E$-configuration in more details. They afforded different photo behavior depending on the position of halogen atom on the benzene ring: while $m$-halonitrosobenzene dimers do not dissociate under UV irradiation, dimeric $p$-chloronitrosobenzene, as well as previously studied $p$-bromonitrosobenzene photolyse very efficiently with recovering of the original crystal phase. Nonreactivity of meta dimers is explained by hindering the necessary rotation of benzene rings caused by interhalogen bonds formed in the crystal lattice. From the kinetics of thermal dimerization measured in solid-state, which afforded typical sigmoid curve, it was found that the process progress as a two-dimensional growth of the product phase.

Acknowledgements. We gratefully acknowledge the financial support to this work from the Ministry of Science, Education, and Sports of the Republic of Croatia, No 119-1191342-1334.

\section{REFERENCES}

1. (a) Th. A. J. Wajer and Th. J. De Boer, Recueil 91 (1972) 565; (b) F. D. Greene and K. E. Gilbert, J. Org. Chem. 40 (1975) 1409; (c) M. L. Greer, H. Sarker, M. E. Medicino, and S. C. Blackstock, J. Am. Chem. Soc. 117 (1995) 10460; (d) B. G. Gowenlock and W. Luttke, Quart. Rev. 12 (1958) 321; (e) J. P. Snyder, M. H. Heyman, and E. Suciu, J. Org. Chem. 40 (1975) 1395; (f) K. G. Orrell, V. Sik, and D. Stephenson, Magn. Reson. Chem. 25 (1987) 1007; (g) K. G. Orrell, D. Stephenson, and T. Rault, Magn. Reson. Chem. 27 (1989) 368; (h) D. A. Fletcher, B. G. Gowenlock, K. G. Orrell, and V. Sik, Magn. Reson. Chem. 33 (1995) 561; (i) D. A. Fletcher, B. G. Gowenlock, and K. G. Orrell, J. Chem. Soc. Perkin Trans 2 (1998) 797; (j) D. A. Fletcher, B. G. Gowenlock, K. G. Orrell, V. Sik, D. E. Hibbs, M. B. Hursthouse, and A. K. M. Malik, J. Chem. Soc., Perkin Trans 2 (1996) 191; (k) B. G. Gowenlock and G. B. Richter-Addo, Chem. Soc. Rev. 34 (2005) 797; (1) D. A. Fletcher, B. G. Gowenlock, and K. G. Orrell, J. Chem. Soc., Perkin Trans. 2 (1997) 2201; (m) M. Azoulay and E. Fischer, J. Chem. Soc., Perkin Trans. 2 (1982) 637.

2. H. Vančik, V. Šimunić-Mežnarić, I. Ćaleta, E. Meštrović, and S. Milovac, J. Phys. Chem. 106 (2002) 1576.

3. (a) H. Vančik, V. Šimunić-Mežnarić, E. Meštrović, and I. Halasz, J. Org. Chem. 69 (2004) 4829; (b) I. Halasz, E. Meštrović, H. Čičak, Z. Mihalić, and H. Vančik, J. Org. Chem. 70 (2005) 8461.

4. (a) K. G. Orrell, D. Stephenson, and J. H. Velarque, J. Chem. Soc. Perkin Trans 2 (1990) 1297; (b) B. S. Furniss, A. J. Hannaford, P. W. G. Smith, and A. R. Tatchell, Vogel's Textbook of Practical Organic Chemistry, Longman Scientific \& Tehnical, London, (1989) 956; (c) B. Priewisch and K. Rück-Braun, J. Org. Chem. 70(6) (2005) 2350.

5. (a) M. Azoulay and E. Fischer, J. Chem. Soc., Perkin Trans. 2 (1982) 637; (b) B. G. Gowenlock, M. J. Maidment, K. G. Orrell, I. Prokeš, and J. R. Roberts, J. Chem. Soc., Perkin Trans. 2 (2001) 1904.

6. 6. (a) M. Avrami, J. Chem. Phys. 8 (1940); (b) Ibid. 9 (1941) 177; (c) B. V. Erofeev, Compt. Rend. Acad. Sci.USSR, 52 (1946) 511; (d) J. D. Hancock and J. H. Sharp, J. Am. Ceram. Soc. 55 (1972) 74 .

7. P. Auffinger, F. A. Hays, E. Westhof, and P. Shing Ho, Proc. Natl. Acad. USA 101(48) (2004) 16789. 